\title{
Sleep, eating disorder symptoms, and daytime functioning
}

This article was published in the following Dove Press journal:

Nature and Science of Sleep

18 January 2016

Number of times this article has been viewed

\author{
Marilou DP Tromp' \\ Anouk AMT Donners' \\ Johan Garssen ${ }^{1,2}$ \\ Joris C Verster ${ }^{1,3}$
}

'Division of Pharmacology, Utrecht Institute for Pharmaceutical Sciences, Utrecht University, Utrecht, the Netherlands; ${ }^{2}$ Nutricia Research, Utrecht, the Netherlands; ${ }^{3}$ Center for Human Psychopharmacology, Swinburne University, Melbourne, VIC, Australia
Correspondence: Joris C Verster Division of Pharmacology, Utrecht Institute for Pharmaceutical Sciences, Utrecht University, Universiteitsweg 99, 3584CG Utrecht, the Netherlands $\mathrm{Tel}+3 \mid 302536909$

Fax +3I 302537900

Email j.c.verster@uu.nl
Objective: To investigate the relationship between eating disorders, body mass index (BMI), sleep disorders, and daytime functioning.

Design: Survey.

Setting: The Netherlands.

Participants: N=574 Dutch young adults (18-35 years old).

Measurements: Participants completed a survey on eating and sleep habits including the Eating Disorder Screen for Primary care (ESP) and SLEEP-50 questionnaire subscales for sleep apnea, insomnia, circadian rhythm disorder (CRD), and daytime functioning. SLEEP-50 outcomes of participants who screened negative $(\leq 2)$ and positive $(>2)$ on the ESP were compared. In addition, SLEEP-50 scores of groups of participants with different ESP scores (0-4) and different BMI groups (ie, underweight, healthy weight, overweight, and obese) were compared using nonparametric statistics.

Results: Almost $12 \%(\mathrm{n}=67)$ of participants screened positive for having an eating disorder. Relative to participants without eating disorders, participants who screened positive for eating disorders reported significantly higher scores on sleep apnea (3.7 versus $2.9, P=0.012$ ), insomnia (7.7 versus 5.5, $P<0.0001)$, CRD (2.9 versus 2.3, $P=0.011$ ), and impairment of daytime functioning ( 8.8 versus $5.8, P=0.0001)$. ESP scores were associated with insomnia ( $r=0.117, P=0.005)$, sleep apnea $(r=0.118, P=0.004)$, sleep quality $(r=-0.104, P=0.012)$, and daytime functioning ( $r=0.225, P<0.0001)$, but not with CRD ( $r=0.066, P=0.112)$. BMI correlated significantly with ESP scores $(r=0.172, P<0.0001)$ and scores on sleep apnea $(r=0.171$, $P<0.0001)$. When controlling for BMI, the partial correlation between ESP and sleep apnea remained significant $(r=0.10, P=0.015)$.

Conclusion: Participants who score positive for eating disorders scored significantly higher on sleep disorder scales, and reported significantly more impairment of daytime functioning.

Keywords: eating disorders, sleep disorders, insomnia, apnea, circadian rhythm disorder, daytime functioning

\section{Introduction}

Both good sleep and caloric intake are essential for survival and health. Sleep disorders are often comorbid with psychiatric diseases, and both may significantly affect daytime functioning and quality of life. ${ }^{1}$ Eating disorders (EDs), including anorexia nervosa, bulimia nervosa, binge eating disorder, and night eating syndrome (NES), are relatively rare when compared to other psychiatric diseases. It is estimated that $1 \%-2 \%$ of the population suffer from EDs, but prevalence rates of up to $5 \%$ have been reported. ${ }^{2}$

The literature on EDs includes reports on the relation between EDs and other psychiatric disorders such as depression and anxiety, ${ }^{3}$ but relatively few studies have 
investigated the potential relationship between EDs and sleep disorders. Addressing this gap in the literature may be important because sleep disorders have long been reported in individuals with EDs. ${ }^{4}$ Most research on the relationship between EDs and sleep has focused on NES or sleep-related ED, which are distinct from daytime EDs. ${ }^{3}$ Studies have shown a relationship between night ED and sleep apnea, ${ }^{5}$ restless legs syndrome, ${ }^{6}$ insomnia, ${ }^{7}$ and sleepwalking. ${ }^{8}$ Results of these studies, however, are often difficult to interpret and various moderating factors allow for different interpretation of the observed associations. For example, Yeh and Brown ${ }^{9}$ found that overweight people were more likely to binge eat before falling asleep, but argued that their results may indicate that eating itself, rather than weight gain or being obese, may actually cause the sleep deficits.

Regarding other EDs, research often examines sleep quality and duration of sleep in patients with anorexia nervosa, bulimia nervosa, and binge ED. ${ }^{10}$ For example, Lacey et a ${ }^{11}$ showed that during successful treatment of ten inpatients with anorexia nervosa (a 100-day hospital stay), significant weight gain was accompanied by a significant increase in total sleep time ( \pm 45 minutes) and a reduction in early morning awakenings. Polysomnographic assessments revealed smallto-moderate changes in electroencephalography sleep patterns in patients with EDs. Relative to healthy controls, patients with EDs showed a reduction of slow-wave sleep and an increased number and duration of nightly awakenings ${ }^{12}$ and changes in total sleep time. ${ }^{13}$ These findings are in line with self-reported reduced sleep quality by patients with EDs. ${ }^{14}$

Several studies focused on the relationship between body mass index (BMI) and sleep disorders. Although not per se related to EDs, BMI is often used to classify subjects as being underweight, having a healthy weight, overweight, or obese. For example, an inverse relationship between BMI and total sleep time was found, and sleep latency was significantly shortened in young adults with greater BMI. ${ }^{15}$ These studies suggest that disordered eating behaviors, including binge eating, may be overrepresented in overweight and obese individuals. Also, a relationship between obesity and the likelihood of sleep apnea has been reported. ${ }^{16}$ Individuals with obesity and sleep apnea report poorer sleep quality and subsequently often report daytime sleepiness. ${ }^{17}$ This in turn may negatively affect quality of life and impair performance of daily activities. For example, people with sleep apnea are more likely to have car crashes than people without sleep disorders. ${ }^{18,19}$ However, it should be noted that many patients with EDs have a BMI that is within the normal limits, and obesity itself generally is unrelated to having an ED.
Hence, EDs are seen across the weight spectrum, and it is therefore unlikely that associated sleep disorders can be fully explained by differences in BMI.

Recent studies examined the relationship between sleep, weight status, and eating habits in the general population. In a 6-year longitudinal study, Chaput et $\mathrm{al}^{20}$ demonstrated that higher scores on a binge-eating scale significantly increased the risk of overeating and weight gain in those with short sleep duration. In another study looking at undergraduate students, scores on the Eating Attitudes Test-40 questionnaire were significantly related to scores on two questions on difficulties of initiating and maintaining sleep. ${ }^{21,22}$ Unfortunately, the presence of specific sleep disorders such as insomnia was not verified by screening questionnaires or formal diagnosis. Another study revealed that 42 out of 65 patients with EDs (64.4\%) reported 'sleeping poorly'. Also in this study, no screening questionnaire or formal diagnosis of sleep disorders were conducted..$^{23}$

Taken together, although a relationship between EDs and sleep disorders has been shown, most research did not actually screen or diagnose for specific sleep disorders. Therefore, the primary aim of this study was to investigate the relationship between ED symptoms and the presence and severity of specific sleep disorders, including sleep apnea, circadian rhythm disorder (CRD), and insomnia. Additionally, this study will assess the effect that EDs and sleep disorders have on daytime functioning.

\section{Methods}

Dutch young adults, 18-35 years old completed a survey collecting data on demographics, eating habits, sleep quality, and sleep duration. Participants were approached to participate at and near the campus of Utrecht University, the Netherlands. They were informed about the purpose of the survey and gave verbal informed consent before completion of the survey, which was administered in-person or online through thesistools.com. There was no reward for participating in the survey. No recruitment rate was recorded, but the vast majority of subjects were willing to participate. The Medical Ethical Review Board advised that no formal approval was required to conduct this survey as per their guidelines.

Demographic data included age, sex, height, and weight. Self-reported weight and height enabled computation of BMI (weight in kilograms divided by the square of the height in meters). According to their BMI, subjects were classified (WHO International Classification) as being underweight (BMI: <18.5), having a healthy weight (BMI: 18.5-24.99), being overweight (BMI: 25.0-29.99), or obese (BMI: >30). 
The Eating Disorder Screen for Primary Care (ESP) was used to screen for EDs. ${ }^{24}$ This screening instrument consists of four questions that can be answered by either "yes" or "no" (Table 1). Answering abnormal to more than two questions indicates a positive screen result for having EDs. A "no" to question 1 and a "yes" to question 2, 3, or 4 was classified as an abnormal response. Participants were classified without an ED if the sum of the ESP score was $\leq 2$. An ESP score $>2$ is a positive screen for having EDs and warrants further investigation and diagnosis of the subject. Cotton et $\mathrm{al}^{24}$ found that using this cutoff results in a sensitivity of $100 \%$ with a corresponding specificity of $71 \%$. Hence, a score $>2$ warrants further investigation of the subject to confirm the actual presence of EDs.

To examine the presence and severity of subjective sleep complaints over the past 4 weeks, four of the nine subscales (26 items) of the Dutch SLEEP-50 questionnaire were completed. ${ }^{25}$ The SLEEP-50 was designed by Spoormaker et al, ${ }^{25}$ who followed the descriptions and criteria from the Diagnostic and Statistical Manual of Mental Disorders, 4th Editon, leading to 50 questions that can be categorized in nine subscales. The four subscales used in the current study were sleep apnea (eight items), insomnia (nine items), CRD (three items), and impact of sleep complaints on daytime functioning (six items). The sleep apnea, insomnia, and CRD subscales were selected because according to the literature, these sleep disorders were most likely to be related to EDs or BMI. Because sleep disorders can have a profound impact on daytime functioning, the corresponding subscale was included as well. Each question was scored on a 4-point scale: 1 (not at all), 2 (somewhat), 3 (rather much), and 4 (very much). In addition to the SLEEP-50 questionnaire subscales, the prior night's total sleep time was recorded, and an overall sleep quality score ranging from 0 (very poor) to 10 (very good) was obtained. The questions on sleep quality and sleep duration are part of the SLEEP-50 questionnaire. Cutoff values for a positive screen of sleep disorders include $\geq 15$ on sleep apnea items (sensitivity $=85 \%$, specificity $=88 \%$ ), $\geq 19$ on insomnia

Table I Answers to the ESP

\begin{tabular}{lll}
\hline & No (\%) & Yes (\%) \\
\hline I. Are you satisfied with your eating patterns? & 26.3 & 73.7 \\
$\begin{array}{l}\text { 2. Do you ever eat in secret? } \\
\text { 3. Does your weight affect the way you feel }\end{array}$ & 70.0 & 30.0 \\
$\begin{array}{l}\text { about yourself? } \\
\text { 4. Do you currently suffer with or have you ever } \\
\text { suffered in the past with an eating disorder? }\end{array}$ & 93.2 & 67.1 \\
\hline
\end{tabular}

Abbreviation: ESP, Eating Disorder Screen for Primary Care. items (sensitivity $=71 \%$, specificity $=75 \%$ ), $\geq 8$ on CRD item (sensitivity $=83 \%$, specificity $=69 \%$ ), and $\geq 15$ on daytime functioning (sensitivity $=84 \%$, specificity $=77 \%$ ). ${ }^{25}$

Finally, subjects reported whether or not they suffered from uncontrolled nocturnal eating episodes, scored on a 4-point scale: 1 (not at all), 2 (somewhat), 3 (rather much), and 4 (very much). This item, which is part of the SLEEP-50 questionnaire, is not used to screen for night EDs, but was included in the survey to collect further information on the relationship between sleep and eating behaviors.

Statistical analyses were conducted using the Statistical Package for the Social Sciences (SPSS) version 20 (IBM SPSS Statistics; IBM Corporation, Armonk, NY, USA). Mean and standard deviation (SD) were computed for each variable and it was determined if scores were normally distributed. If the data were not normally distributed, appropriate nonparametric tests were used for the statistical analyses.

SLEEP-50 questionnaire subscale scores of subjects who screened positive for ED (ESP score $>2$ ) were compared to those who scored negative for ED (ESP score $\leq 2$ ), using an independent samples Kruskal-Wallis test. SLEEP-50 questionnaire subscale scores of groups of subjects with different ESP scores $(1,2,3$, or 4) were compared with the group of subjects having an ESP score of zero, using an independent samples Mann-Whitney $U$-test. Results were considered significant if $P<0.05$.

SLEEP-50 questionnaire scores of the subjects of different BMI groups (underweight, overweight, and obese) were compared with subjects of the BMI group with a healthy weight using an independent samples Kruskal-Wallis test. Also, the percentage of subjects who scored positive for EDs was computed for each BMI group. Finally, the association between BMI, ESP scores, sleep quality, and SLEEP-50 questionnaire scores was assessed. As the data was not always normally distributed, this was done with nonparametric correlational analyses (Spearman's $r$ ).

\section{Results Participant characteristics}

There were 581 participants that completed the survey. Seven participants were excluded because of recent surgery $(n=1)$, irregular sleep patterns due to shiftwork $(n=3)$, or because they were younger than 18 years of age $(n=3)$. Thus data from 574 participants were included in the statistical analyses. The mean (SD) age was 22.2 (3.1) years, and 68.5\% were women. Of the total sample, $n=67(11.7 \%)$ participants screened positive for having an ED. Fourteen (2.4\%) participants reported having uncontrolled nocturnal eating episodes. On average, 
subjects rated their sleep quality at 7.1 (1.2) on a scale from 0 (very poor) to 10 (very good), and reported a mean (SD) sleep duration of 7.6 (1.1) hours. Only $2.3 \%$ of the participants met the SLEEP-50 criteria as having a sleep disorder: for insomnia $(n=9)$, sleep apnea $(n=1)$, or CRD $(n=3)$.

\section{Relationship between ESP and SLEEP-50 scores}

Results from the ESP are summarized in Table 1.

Significant correlations were found between ESP scores and scores on insomnia ( $r=0.117, P=0.005)$, sleep apnea ( $r=0.118, P=0.004)$ daytime functioning $(r=0.225$, $P<0.0001)$, and sleep quality $(r=-0.104, P=0.012)$, but not for CRD ( $r=0.066, P=0.112$ ). Correlations between daytime functioning and insomnia $(r=0.415, P=0.0001), \mathrm{CRD}$ ( $r=0.451, P=0.0001)$, sleep apnea $(r=0.289, P<0.0001)$, and sleep quality $(r=-0.435, P<0.0001)$ were also significant, and were stronger than those observed between ESP and sleep disorders. When controlling for scores on the SLEEP-50 subscales of insomnia, sleep apnea, and CRD, the partial correlation between ESP and daytime functioning scores was somewhat less pronounced, but remained significant ( $r=0.188, P \leq 0.0001)$. ESP scores and total sleep time were negatively correlated $(r=-0.092, P=0.028)$. Total sleep time was significantly shorter in those who screened positive for having EDs (7.3 versus 7.6 hours, $P=0.034$ ). Also, a clear difference in sleep disorder scores was found between those who screened positive and negative for ED (Table 2).

Table 2 SLEEP-50 subscale scores for participants who score positive $(>2)$ and negative $(\leq 2)$ on the ESP

\begin{tabular}{lll}
\hline SLEEP-50 scale & ESP positive & ESP negative \\
\hline Sleep apnea & & \\
$\quad$ Mean (SD) & $3.7(2.9)$ & $2.9(2.3)$ \\
Median & 3.0 & 2.0 \\
IQR & 3.0 & 3.0 \\
Insomnia & & \\
Mean (SD) & $7.7(5.2)$ & $5.5(4.1)$ \\
Median & 7.0 & 5.0 \\
IQR & 7.0 & 6.0 \\
Circadian rhythm disorder & & \\
Mean (SD) & $2.9(2.0)$ & $2.3(1.8)$ \\
Median & 3.0 & 2.0 \\
IQR & 3.0 & 2.0 \\
Impairment of daytime functioning & \\
Mean (SD) & $8.8(4.1)$ & $5.8(4.0)$ \\
Median & 8.0 & 5.0 \\
IQR & 5.0 & 7.0 \\
\hline
\end{tabular}

Abbreviations: ESP, Eating Disorders Screen for Primary Care; SD, standard deviation; IQR, interquartile range.
Participants who screened positive for ED reported significantly higher scores on sleep apnea (3.7 versus 2.9, $P=0.017)$, insomnia ( 7.7 versus $5.5, P=0.001)$, CRD (2.9 versus $2.3, P=0.005)$, impairment of daytime functioning (8.8 versus 5.8, $P<0.0001)$, sleep quality $(P<0.0001)$, and total sleep time $(P=0.036)$ relative to participants screening negative for ED. Figure 1 shows the increment of SLEEP-50 scores for each ESP score, relative to subjects with an ESP score of zero. Significant differences $(P<0.05)$ are indicated by asterisks.

\section{Relationship between BMI and SLEEP-50 scores}

The majority of participants had a BMI that fell in the normal range $(n=487,84.8 \%)$. Fifty-eight $(10.1 \%)$ participants were overweight, $8(1.4 \%)$ were obese, and 21 (3.7\%) were underweight. SLEEP-50 scores on sleep apnea, insomnia, CRD, and daytime functioning did not differ significantly among the BMI groups. BMI scores were significantly correlated with scores on sleep apnea $(r=0.171, P<0.0001)$, but not with other sleep disorders, sleep quality, and daytime functioning. When controlling for BMI, the partial correlation between ESP and sleep apnea remained significant $(r=0.10, P=0.015)$. BMI was significantly correlated with ESP scores $(r=0.172, P<0.0001)$. Total sleep time was correlated negatively with BMI $(r=-0.137$, $P=0.001)$. No significant differences among BMI groups were observed for sleep quality scores and total sleep time.

\section{Discussion}

Our data suggest that individuals who screen positive for EDs commonly experience sleep disorders such as insomnia and sleep apnea. Impairment of daytime functioning was more pronounced in participants who scored higher on the ESP and SLEEP-50 subscales. These findings confirm and extend previous research. ${ }^{12-14}$

It may be worthwhile to screen ED patients for possible sleep disorders. The observed relationship between EDs and sleep disorders may have implications for treatment of patients with EDs. It should be established by future research whether changing sleep habits (eg, improving sleep hygiene) or treating sleep disorders has a positive effect on the treatment of EDs. There is already a vast body of literature indicating that cognitive behavioral therapy is helpful or effective in treatment of sleep disorders such as insomnia. ${ }^{26}$ Future research should investigate whether a specific focus on sleep habits during cognitive behavioral therapy or acceptance and commitment therapy has a positive impact on ED psychopathology. 
Insomnia

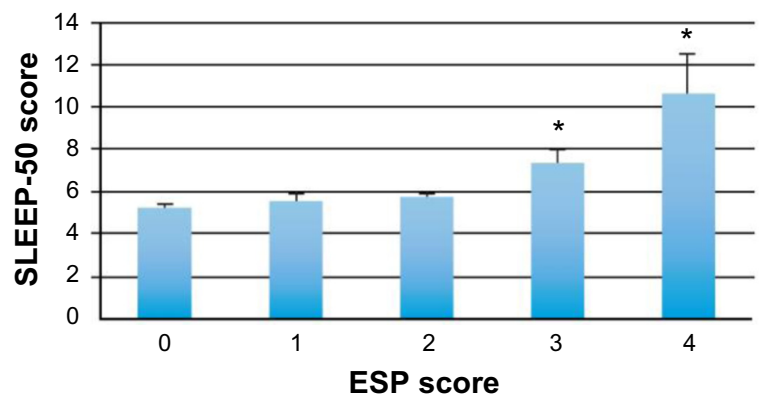

Daytime functioning

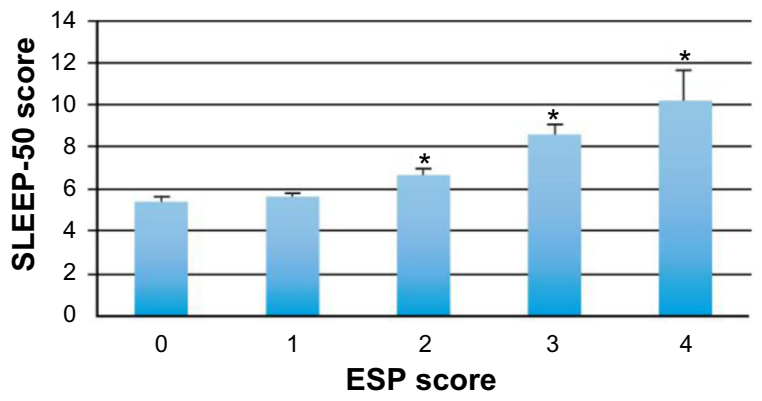

Sleep apnea

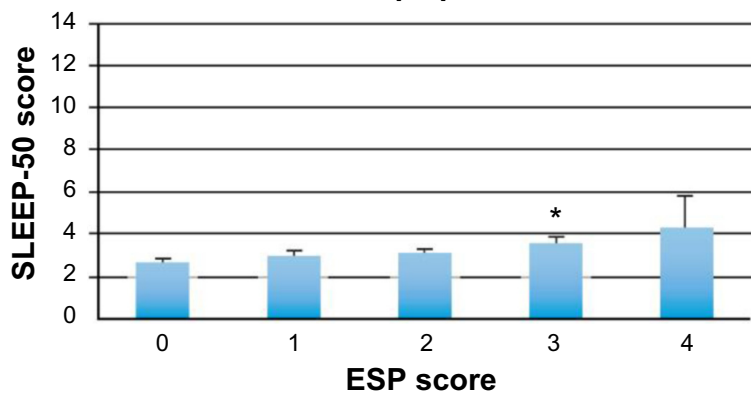

Circadian rhythm disorder

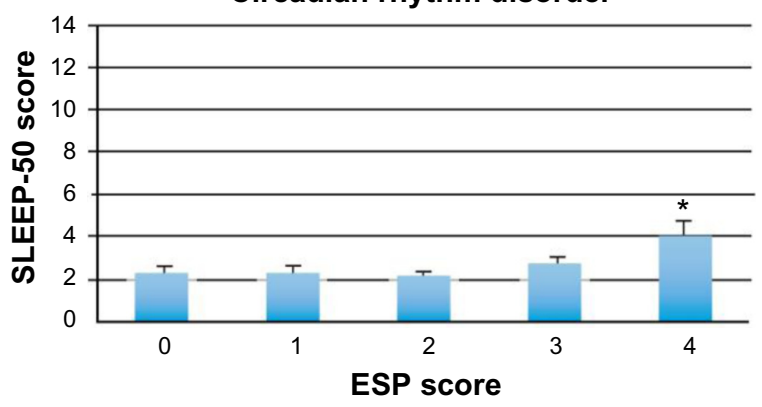

Figure I Relationship between SLEEP-50 scores and ESP scores.

Notes: Mean (SD) SLEEP-50 scores are shown for groups of people with different ESP scores (0-4). An ESP score $>2$ is a positive screen for having eating disorders and warrants further investigation of the subject. Significant differences from the group with an ESP score of zero $(P<0.05)$ are indicated by *.

Abbreviations: ESP, Eating Disorder Screen for Primary Care; SD, standard deviation.

Previous literature on the relationship between daytime ED and sleep disorders is relatively small, and surprisingly, generally does not include screening for, or formal diagnosis of, sleep disorders such as sleep apnea and insomnia. Inclusion of the SLEEP-50 subscales in the current study can therefore be regarded as a strength relative to previous work on this topic. Several limitations, however, should also be addressed. Most importantly, both the ESP and SLEEP-50 questionnaire are screening instruments that are not intended to establish a formal diagnosis. This should be taken into account when interpreting the results. However, the scales indicate that certain subjects are potentially at risk of having an ED and/or sleep disorder. Given the observed associations between SLEEP-50 and ESP scores, further investigation is warranted. Future experimental studies should include formal diagnosis of sleep and EDs that allows differentiation between the various subtypes. Supervised sleep, including polysomnography, can further help to objectively measure sleep disorders.

In this regard, it should also be noted that the ESP does not differentiate between different types of EDs (eg, anorexia, bulimia, or NES). However, it can be expected that different types of EDs may be differently associated with insomnia, sleep apnea, and CRDs. Future research should therefore include validated specific scales to identify the risk of the various types of EDs.

The current study included young adults recruited at Utrecht University. Although generalizability of (prevalence) data from a convenience sample is often regarded as problematic, this is of less concern when investigating associations. Nevertheless, the age range of our study is small, and as most participants were students, it can be questioned whether our sample is representative for the general population as a whole. Hence, this study should be replicated in a nonstudent population; recruiting participants from a wider age range, and preferably also include a group of participants who are formally diagnosed as having an ED. It then may also be of interest to differentiate between participants having different EDs, which is not possible when using the ESP screening questionnaire, and determine whether participants may have comorbid mood disorders such as anxiety and depression that may also affect sleep. Finally, the subjective nature (self-report) of the data and the risk of recall bias are inherent to survey research. Hence, no causal relationships can be drawn from our data. Other factors affecting health status such as smoking and drinking alcohol all may play a role in the relationship between EDs and sleep disorders. Our survey should therefore be considered as a pilot study providing potential leads for future research.

Notwithstanding the explorative nature of our study, we found a positive association between EDs and sleeping disorders scores. Participants who score positive for EDs scored significantly higher on sleep disorder scales, and reported 
significantly more impairment of daytime functioning. The relationship between sleep disorders and BMI was less clear. Future studies including formal diagnosis of sleep disorders and EDs should be conducted to confirm our findings.

\section{Disclosure}

This study was supported by Utrecht University.

Joris Verster has received grants/research support from The Dutch Ministry of Infrastructure and the Environment, Janssen Research and Development, Nutricia Research, Takeda, Red Bull and has acted as a consultant for the Canadian Beverage Association, Centraal Bureau Drogisterijbedrijven, Coleman Frost, Danone Research, Deenox, Eisai, Jazz, Purdue, Red Bull, Sanofi-Aventis, Sepracor, Takeda, Transcept, and Trimbos Institute. Johan Garssen is a part time employee of Nutricia Research. The authors report no other conflicts of interest in this work.

\section{References}

1. Verster JC, Pandi-Perumal SR, Streiner DL, editors. Sleep and Quality of Life in Clinical Medicine. Totowa, NJ: Humana Press (Springer); 2008.

2. Smink FR, van Hoeken D, Hoek HW. Epidemiology of eating disorders: incidence, prevalence and mortality rates. Curr Psychiatry Rep. 2012;14: 406-414.

3. Van der Wal JS. Night eating syndrome: a critical review of the literature. Clin Psychol Rev. 2012;32:49-59.

4. Crisp AH, Stonehill E, Fenton GW. The relationship between sleep, nutrition and mood: a study of patients with anorexia nervosa. Postgrad Med J. 1971;47:207-213.

5. Olbrich K, Mühlhans B, Allison KC, et al. Night eating, binge eating and related features in patients with obstructive sleep apnea syndrome. Eur Eat Disord Rev. 2009;17:120-127.

6. Antelmi E, Vinai P, Pizza F, et al. Nocturnal eating is part of the clinical spectrum of restless legs syndrome and an underestimated risk factor for increased body mass index. Sleep Med. 2014;15:168-172.

7. Palmese LB, DeGeorge PC, Ratliff JC, et al. Insomnia is frequent in schizophrenia and associated with night eating and obesity. Schizophr Res. 2011;133:238-243.

8. Schenk CH, Mahowald MW. Review of nocturnal sleep-related eating disorders. Int J Eat Disord. 1994;15:343-356.
9. Yeh SS, Brown RF. Disordered eating partly mediates the relationship between poor sleep quality and high body mass index. Eat Behav. 2014:15:291-297.

10. Lundgren JD, O'Reardon JP, Alliston KC, et al. Sleep and Quality of Life in Eating Disorders. In: Verster JC, Pandi-Perumal SR, Streiner DL, editors. Sleep and Quality of Life in Clinical Medicine. Totowa, NJ: Humana Press (Springer); 2008:281-289.

11. Lacey JH, Crisp AH, Kalucy RS, et al. Weight gain and the sleeping electroencephalogram: study of 10 patients with anorexia nervosa. $B M J$. 1975;4:556-558.

12. Lauer CJ, Krieg JC. Sleep in eating disorders. Sleep Med Rev. 2004;8: 109-118.

13. Tzischinsky O, Latzer Y, Epstein R, et al. Sleep-wake cycles in women with binge eating disorder. Int J Eat Disord. 2000;27:43-48.

14. Vardar E, Caliyurt O, Arikan E, et al. Sleep quality and psychopathological features in obese binge eaters. Stress Health. 2004;20:35-41.

15. Meyer KA, Wall MM, Larson N, et al. Sleep duration and BMI in a sample of young adults. Obesity. 2012;20:1279-1287.

16. Sloan EP, Shapiro CM. Obstructive sleep apnea in a consecutive series of obese women. Int J Eat Disord. 1995;17:167-173.

17. Panossian LA, Veasey SC. Daytime sleepiness in obesity: mechanisms beyond obstructive sleep apnea - a review. Sleep. 2012;35:605-615.

18. Rodenstein DO, Alonderis A, Auwaerts G, et al. Driving in Europe: the need of a common policy for drivers with obstructive sleep apnoea syndrome. J Sleep Res. 2008;17:281-284.

19. Pizza F, Contardi S, Mondini S, et al. Daytime sleepiness and driving performance in patients with obstructive sleep apnea: comparison of the MSLT, the MWT, and a simulated driving task. Sleep. 2009;32: 382-391.

20. Chaput JP, Després JP, Bouchard C, et al. The association between short sleep duration and weight gain is dependent on disinhibited eating behavior in adults. Sleep. 2011;34:1291-1297.

21. Boss SC, Soares MJ, Marques M, et al. Disordered eating behaviors and sleep disturbances. Eat Behav. 2013;14:192-198.

22. Soares MJ, Macedo A, Bos SC, et al. Sleep disturbances, body mass index and eating behaviour in undergraduate students. $J$ Sleep Res. 2011;20:479-486

23. Ross C, Herman PM, Rocklin O, et al. Evaluation of integrative medicine supplements for mitigation of chronic insomnia and constipation in an inpatient eating disorders setting. Explore (NY). 2008;4:315-320.

24. Cotton MA, Ball C, Robinson P. Four simple questions can help screen for eating disorders. J Gen Intern Med. 2003;18:53-56.

25. Spoormaker VI, Verbeek I, van den Bout J, et al. Initial validation of the SLEEP-50 questionnaire. Behav Sleep Med. 2005;3:227-246.

26. Ho FY, Chung KF, Yeung WF, et al. Self-help cognitive-behavioral therapy for insomnia: a meta-analysis of randomized controlled trials. Sleep Med Rev. 2015;19:17-28.
Nature and Science of Sleep

\section{Publish your work in this journal}

Nature and Science of Sleep is an international, peer-reviewed, open access journal covering all aspects of sleep science and sleep medicine, including the neurophysiology and functions of sleep, the genetics of sleep, sleep and society, biological rhythms, dreaming, sleep disorders and therapy, and strategies to optimize healthy sleep. The journal welcomes
Dovepress

original research, clinical \& epidemiological studies, reviews \& evaluations, case reports and extended reports. The manuscript management system is completely online and includes a very quick and fair peerreview system, which is all easy to use. Visit http://www.dovepress.com/ testimonials.php to read real quotes from published authors. 